\title{
Integral trees of arbitrarily large diameters
}

\author{
Péter Csikvári
}

Received: 21 September 2009 / Accepted: 31 January 2010 / Published online: 18 February 2010

(C) Springer Science+Business Media, LLC 2010

\begin{abstract}
In this paper, we construct trees having only integer eigenvalues with arbitrarily large diameters. In fact, we prove that for every finite set $S$ of positive integers there exists a tree whose positive eigenvalues are exactly the elements of $S$. If the set $S$ is different from the set $\{1\}$ then the constructed tree will have diameter $2|S|$.
\end{abstract}

Keywords Trees $\cdot$ Eigenvalues

\section{Introduction}

An integral tree is a tree for which the eigenvalues of its adjacency matrix are all integers [9]. Many different classes of integral trees have been constructed in the past decades [2-5, 10-12]. Most of these classes contain infinitely many integral trees, but till now only examples of trees of bounded diameters were known. The largest diameter of known integral trees was 10. In this paper, we construct integral trees of arbitrarily large diameters. In fact, we prove the following much stronger theorem.

Theorem 1.1 For every finite set $S$ of positive integers there exists a tree whose positive eigenvalues are exactly the elements of $S$. If the set $S$ is different from the set $\{1\}$ then the constructed tree will have diameter $2|S|$.

Clearly, there is only one tree with set $S$ of positive eigenvalues for $S=\{1\}$, the tree on two vertices with spectrum $\{-1,1\}$ (and its diameter is 1 ).

To the memory of Gács András.

P. Csikvári (凶)

Department of Computer Science, Eötvös Loránd University, Pázmány Péter sétány 1/C, 1117

Budapest, Hungary

e-mail: csiki@cs.elte.hu 
The structure of this paper is the following. In the next section, we will define a class of trees recursively. All trees belonging to this class will turn out to be almostintegral, i.e., all of their eigenvalues are square roots of integers. We will find integral trees in this class of trees by special choice of parameters introduced later.

\section{Construction of trees}

Definition 2.1 For given positive integers $r_{1}, \ldots, r_{k}$ we construct the trees $T_{1}\left(r_{1}\right)$, $T_{2}\left(r_{1}, r_{2}\right), \ldots, T_{k}=T_{k}\left(r_{1}, \ldots, r_{k}\right)$ recursively as follows. We will consider the tree $T_{i}$ as a bipartite graph with color classes $A_{i-1}, A_{i}$. The tree $T_{1}\left(r_{1}\right)=\left(A_{0}, A_{1}\right)$ consists of the classes of size $\left|A_{0}\right|=1,\left|A_{1}\right|=r_{1}$ (so it is a star on $r_{1}+1$ vertices). If the tree $T_{i}\left(r_{1}, \ldots, r_{i}\right)=\left(A_{i-1}, A_{i}\right)$ is defined then let $T_{i+1}\left(r_{1}, \ldots, r_{i+1}\right)=\left(A_{i}, A_{i+1}\right)$ be defined as follows. We connect each vertex of $A_{i}$ with $r_{i+1}$ new vertices of degree 1 . Then for the resulting tree the color class $A_{i+1}$ will have size $\left|A_{i+1}\right|=r_{i+1}\left|A_{i}\right|+$ $\left|A_{i-1}\right|$, the color class $A_{i}$ does not change. (See Fig. 1.)

One should not confuse these trees with the balanced trees. These trees are very far from being balanced.

Fig. 1 Let $A_{i+1}=A_{i-1} \cup B_{i}$ where each element of $A_{i}$ has exactly $r_{i+1}$ neighbors of degree 1 in $B_{i}$

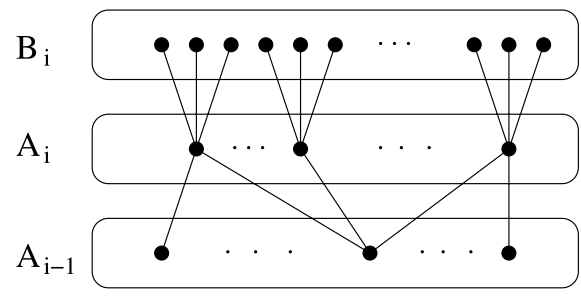

To analyze the trees $T_{k}\left(r_{1}, \ldots, r_{k}\right)$ we will need the following definition.

Definition 2.2 Let us define the following sequence of expressions.

$$
\begin{gathered}
Q_{0}(\cdot)=1, \\
Q_{1}\left(x_{1}\right)=x_{1}
\end{gathered}
$$

and

$$
Q_{j}\left(x_{1}, \ldots, x_{j}\right)=x_{j} Q_{j-1}\left(x_{1}, \ldots, x_{j-1}\right)+Q_{j-2}\left(x_{1}, \ldots, x_{j-2}\right)
$$

for all $3 \leq j \leq k$. We will also use the convention $Q_{-1}=0$. We will call these expression continuants. Sometimes if $\underline{x}=\left(x_{1}, \ldots, x_{k}\right)$ is well-understood then we will simply write $Q_{j}$ instead of $Q_{j}\left(x_{1}, \ldots, x_{j}\right)$.

Remark 2.3 The first few continuants are

$$
Q_{2}\left(x_{1}, x_{2}\right)=1+x_{1} x_{2}, \quad Q_{3}\left(x_{1}, x_{2}, x_{3}\right)=x_{1}+x_{3}+x_{1} x_{2} x_{3},
$$




$$
Q_{4}\left(x_{1}, x_{2}, x_{3}, x_{4}\right)=1+x_{1} x_{2}+x_{1} x_{4}+x_{3} x_{4}+x_{1} x_{2} x_{3} x_{4} .
$$

The expressions $Q_{j}\left(x_{1}, \ldots, x_{j}\right)$ often show up in the study of some Euclidean type algorithms. For instance,

$$
x_{k}+\frac{1}{x_{k-1}+\frac{1}{x_{k-2}+\frac{1}{\ddots \cdot+\frac{1}{x_{1}}}}}=\frac{Q_{k}\left(x_{1}, \ldots, x_{k}\right)}{Q_{k-1}\left(x_{1}, \ldots, x_{k-1}\right)} .
$$

For more details on continuants, see [8].

Lemma 2.4 Let $T_{k}\left(r_{1}, \ldots, r_{k}\right)$ be the constructed tree with color classes $\left(A_{k-1}, A_{k}\right)$. Then $\left|A_{k-1}\right|=Q_{k-1}\left(r_{1}, \ldots, r_{k-1}\right)$ and $\left|A_{k}\right|=Q_{k}\left(r_{1}, \ldots, r_{k}\right)$.

Proof This is a trivial induction.

Lemma 2.5 If $r_{1} \geq 2$ then the diameter of $T_{k}\left(r_{1}, \ldots, r_{k}\right)$ is $2 k$.

Proof Note that each vertex is at a distance of at most $k$ from the only element $v_{0}$ of the set $A_{0}$. Thus the diameter is at most $2 k$. On the other hand, if we go from $v_{0}$ to two different leaves through two different elements of $A_{1}$ which are at a distance $k$ from $v_{0}$ (so these are the elements of $A_{k} \backslash A_{k-2}$ ) then these two leaves must be $2 k$ distance units apart.

Remark 2.6 Note that $T_{j}\left(1, r_{2}, r_{3}, \ldots, r_{j}\right)=T_{j-1}\left(r_{2}+1, r_{3}, \ldots, r_{j}\right)$. Hence all constructed trees different from the tree on two vertices have a representation $T_{k}\left(r_{1}, \ldots, r_{k}\right)$ in which $r_{1} \geq 2$.

The next lemma will be the main tool to determine the spectrum of the tree $T_{k}\left(r_{1}, \ldots, r_{k}\right)$. Before we state it, we introduce the following notation.

Definition 2.7 Let $\operatorname{Sp}(G)$ denote the spectrum of the graph $G$. Let $N_{G}^{+}$denote the number of positive eigenvalues of $G$ and $N_{G}(t)$ denote the multiplicity of the eigenvalue $t$.

Lemma 2.8 Let $G=(A, B, E)$ be a bipartite graph with $\lambda \neq 0$ eigenvalue of multiplicity $m$. Let $G^{\prime}$ be obtained from $G$ by joining each element of $B$ with $r$ new vertices of degree 1 , so that the obtained graph has $|A|+(r+1)|B|$ vertices. Then $\pm \sqrt{\lambda^{2}+r}$ are the eigenvalues of $G^{\prime}$ of multiplicity $m$. Furthermore, the rest of the eigenvalues of the new graph are $\pm \sqrt{r}$ with multiplicity $|B|-N_{G}^{+}$and 0 with multiplicity $|A|+(r-1)|B|$, and there is no other eigenvalue.

Proof Since $G$ and $G^{\prime}$ are both bipartite graphs, we only need to deal with the nonnegative eigenvalues. Let $0<\mu \neq \sqrt{r}$ be an eigenvalue of the graph $G^{\prime}$ of multiplicity $m$. We prove that $\sqrt{\mu^{2}-r}$ is an eigenvalue of $G$ of multiplicity $m$. (Note 
that it means that $0<\mu<\sqrt{r}$ cannot occur since the eigenvalues of a graph are real numbers.)

Let $\underline{x}$ be an eigenvector belonging to $\mu$. We will construct an eigenvector $x^{\prime}$ belonging to $\sqrt{\mu^{2}-r}$ in the graph $G$. Let $v_{i} \in B$ and its new neighbors $w_{i 1}, \ldots, w_{i r}$. Then

$$
x\left(v_{i}\right)=\mu x\left(w_{i 1}\right)=\mu x\left(w_{i 2}\right)=\cdots=\mu x\left(w_{i r}\right) .
$$

Since $\mu \neq 0$ we have $x\left(w_{i 1}\right)=\cdots=x\left(w_{i r}\right)$. Moreover, for each $v_{i} \in B$ and $u_{j} \in A$ we have

$$
\mu x\left(v_{i}\right)=\sum_{v_{i} \sim u_{k}} x\left(u_{k}\right)+r x\left(w_{i 1}\right)
$$

and

$$
\mu x\left(u_{j}\right)=\sum_{u_{j} \sim v_{l}} x\left(v_{l}\right) .
$$

Since $x\left(v_{i}\right)=\mu x\left(w_{i 1}\right)$ we can rewrite these equations as

$$
\left(\mu^{2}-r\right) x\left(w_{i 1}\right)=\sum_{v_{i} \sim u_{k}} x\left(u_{k}\right)
$$

and

$$
\mu x\left(u_{j}\right)=\sum_{u_{j} \sim v_{l}} \mu x\left(w_{l 1}\right) .
$$

In the second equation, we can divide by $\mu$ since it is not 0 . Hence it follows that

$$
\sqrt{\mu^{2}-r} \cdot\left(\sqrt{\mu^{2}-r} \cdot x\left(w_{i 1}\right)\right)=\sum_{v_{i} \sim u_{k}} x\left(u_{k}\right)
$$

and

$$
\sqrt{\mu^{2}-r} \cdot x\left(u_{j}\right)=\sum_{u_{j} \sim v_{l}}\left(\sqrt{\mu^{2}-r} \cdot x\left(w_{l 1}\right)\right) .
$$

Thus the vector $x^{\prime}$ which is equal to $\sqrt{\mu^{2}-r} \cdot x\left(w_{i 1}\right)$ on the vertices of $B$ and $x\left(u_{j}\right)$ on the elements of $A$ is an eigenvector of the graph $G$ with eigenvalue $\sqrt{\mu^{2}-r}$. Clearly, this vector is not $\underline{0}$, otherwise $\underline{x}$ should have been $\underline{0}$. It also implies that if the vectors $\underline{x}_{1}, \ldots, \underline{x}_{h}$ are independent eigenvectors belonging to $\mu$ then the constructed eigenvectors $\underline{x}_{1}^{\prime}, \ldots, \underline{x}_{h}^{\prime}$ are also independent. Note that this construction can be reversed if $\sqrt{\mu^{2}-r} \neq 0$, implying that for $\mu \neq \sqrt{r}$ the multiplicity of $\mu$ in $G^{\prime}$ is the same as the multiplicity of $\sqrt{\mu^{2}-r}$ in $G$.

We can easily determine the multiplicity of the eigenvalues 0 and $\sqrt{r}$ as follows:

$$
\begin{aligned}
e(G)+r|B|=e\left(G^{\prime}\right) & =\sum_{\mu>0, \mu \in S p\left(G^{\prime}\right)} \mu^{2}=\sum_{\lambda>0, \lambda \in S p(G)}\left(\lambda^{2}+r\right)+N_{G^{\prime}}(\sqrt{r}) r \\
& =e(G)+N_{G}^{+} r+N_{G^{\prime}}(\sqrt{r}) r .
\end{aligned}
$$


Hence $N_{G^{\prime}}(\sqrt{r})=|B|-N_{G}^{+}$. Finally, the multiplicity of 0 as an eigenvalue of $G^{\prime}$ can be determined as follows:

$$
\begin{aligned}
N_{G^{\prime}}(0) & =|A|+(r+1)|B|-2 N_{G^{\prime}}^{+} \\
& =|A|+(r+1)|B|-2 N_{G}^{+}-2 N_{G^{\prime}}(\sqrt{r})=|A|+(r+1)|B|-2|B| .
\end{aligned}
$$

Theorem 2.9 The set of different eigenvalues of the tree $T_{k}\left(r_{1}, r_{2}, \ldots, r_{k}\right)$ is the set

$$
\left\{ \pm \sqrt{r_{k}}, \pm \sqrt{r_{k}+r_{k-1}}, \pm \sqrt{r_{k}+r_{k-1}+r_{k-2}}, \ldots, \pm \sqrt{r_{k}+\cdots+r_{1}}, 0\right\}
$$

Furthermore, the multiplicity of 0 is

$$
Q_{k}\left(r_{1}, \ldots, r_{k}\right)-Q_{k-1}\left(r_{1}, \ldots, r_{k-1}\right)
$$

and the multiplicities of the eigenvalues $\pm \sqrt{r_{k}+r_{k-1}+\cdots+r_{j}}$ are

$$
Q_{j-1}\left(r_{1}, \ldots, r_{j-1}\right)-Q_{j-2}\left(r_{1}, \ldots, r_{j-2}\right),
$$

where $Q_{i}$ 's are the continuants.

Proof We will use the short notation $Q_{j}$ for $Q_{j}\left(r_{1}, \ldots, r_{j}\right)$. We prove by induction on $k$. The statement is true for $k=1$. Assume that the statement is true for $n=k-1$. We need to prove it for $n=k$. By the induction hypothesis, the tree $T_{k-1}\left(r_{1}, \ldots, r_{k-1}\right)$ has spectrum

$$
\left\{ \pm \sqrt{r_{k-1}}, \pm \sqrt{r_{k-1}+r_{k-2}}, \ldots, \pm \sqrt{r_{k-1}+\cdots+r_{1}}, 0\right\}
$$

Furthermore, the multiplicities of the eigenvalues $\pm \sqrt{r_{k-1}+\cdots+r_{j}}$ are $Q_{j-1}-$ $Q_{j-2}$. Now let us apply Lemma 2.8 with $G=T_{k-1}\left(r_{1}, \ldots, r_{k-1}\right)$ and $r=r_{k}$. Then $G^{\prime}=T_{k}\left(r_{1}, \ldots, r_{k}\right)$ has spectrum

$$
\left\{ \pm \sqrt{r_{k}}, \pm \sqrt{r_{k}+r_{k-1}}, \pm \sqrt{r_{k}+r_{k-1}+r_{k-2}}, \ldots, \pm \sqrt{r_{k}+\cdots+r_{1}}, 0\right\} .
$$

Furthermore, the multiplicities of the eigenvalues $\pm \sqrt{r_{k}+r_{k-1}+\cdots+r_{j}}$ are $Q_{j-1}-Q_{j-2}$ for $j \leq k-1$. The multiplicity of $\sqrt{r_{k}}$ is

$$
Q_{k-1}-\left(\left(Q_{k-2}-Q_{k-3}\right)+\left(Q_{k-3}-Q_{k-4}\right)+\cdots+\left(Q_{0}-Q_{-1}\right)\right)=Q_{k-1}-Q_{k-2} \text {. }
$$

Finally, the multiplicity of 0 is

$$
\left(r_{k}-1\right) Q_{k-1}+Q_{k-2}=Q_{k}-Q_{k-1}
$$

Remark 2.10 Note that if $r_{1} \geq 2$ then the tree $T_{k}\left(r_{1}, \ldots, r_{k}\right)$ has $2 k+1$ different eigenvalues and diameter $2 k$. Since the number of different eigenvalues is at least the diameter plus one for any graph [7], these trees have the largest possible diameter among graphs having restricted number of different eigenvalues. 
Theorem 1.1 For every set $S$ of positive integers there exists a tree whose positive eigenvalues are exactly the elements of $S$. If the set $S$ is different from the set $\{1\}$ then the constructed tree will have diameter $2|S|$.

Proof Let $S=\left\{n_{1}, n_{2}, \ldots, n_{|S|}\right\}$ where $n_{1}<n_{2}<\cdots<n_{|S|}$. Then apply the previous theorem with

$$
r_{|S|}=n_{1}^{2}, r_{|S|-1}=n_{2}^{2}-n_{1}^{2}, \ldots, r_{1}=n_{|S|}^{2}-n_{|S|-1}^{2} .
$$

If the set is different from $\{1\}$ then $r_{1} \geq 2$, and in this case the diameter of the tree is $2|S|$ by Lemma 2.5 .

Example 1 Let $S=\{1,2,4,5\}$ then $r_{4}=1, r_{3}=3, r_{2}=12, r_{1}=9$. The resulting tree has 781 vertices and the spectrum is

$$
\left\{-5,-4^{8},-2^{100},-1^{227}, 0^{109}, 1^{227}, 2^{100}, 4^{8}, 5\right\} \text {. }
$$

Here the exponents are the multiplicities of the eigenvalues.

Example 2 Let $S=\{1,2,3,4,5,6\}$ then $r_{6}=1, r_{5}=3, r_{4}=5, r_{3}=7, r_{2}=9, r_{1}=$ 11. The resulting tree has 27007 vertices and the spectrum is

$$
\left\{ \pm 6, \pm 5^{10}, \pm 4^{89}, \pm 3^{611}, \pm 2^{2944}, \pm 1^{8021}, 0^{3655}\right\} .
$$

The diameter of this tree is 12 .

Remark 2.11 Recently Andries E. Brouwer (private communication) found a very elegant (and very short!) proof that $T\left(n_{k}^{2}-n_{k-1}^{2}, n_{k-1}^{2}-n_{k-2}^{2}, \ldots, n_{2}^{2}-n_{1}^{2}, n_{1}^{2}\right)$ are integral trees. It is really worth reading this proof. This proof is outlined on Brouwer's homepage [1] or a bit more detailed version of this proof can be found in [6].

Acknowledgement I am very very grateful to András Gács and Andries E. Brouwer for numerous remarks and suggestions.

\section{References}

1. Brouwer, A.E.: http://www.win.tue.nl/ aeb/graphs/integraltrees.html

2. Brouwer, A.E.: Small integral trees. Electron. J. Comb. 15 (2008)

3. Brouwer, A.E., Haemers, W.H.: The integral trees with spectral radius 3. Linear Algebra Appl. 429, 2710-2718 (2008)

4. Cao, Z.F.: Integral trees of diameter $R(3 \leq R \leq 6)$. Heilongjiang Daxue Ziran Kexue Xuebao 95(2), 1-3 (1988)

5. Cao, Z.F.: Some new classes of integral trees with diameter 5 or 6. J. Syst. Sci. Math. Sci. 11, 20-26 (1991)

6. Csikvári, P.: Short note on the integrality of some trees. http://www.cs.elte.hu/ csiki

7. Godsil, C., Royle, G.: Algebraic Graph Theory. Graduate Texts in Mathematics. Springer, Berlin (2001), pp. 186

8. Graham, R.L., Knuth, D.E., Patashnik, O.: Concrete Mathematics. A Foundation for Computer Science, 2nd edn. Addison-Wesley, Reading (1994) 
9. Harary, F., Schwenk, A.J.: Which graphs have integral spectra? In: Graph and Combinatorics. Lecture Notes in Mathematics, vol. 406, pp. 45-51. Springer, Berlin (1974)

10. Wang, L.G., Li, X.L.: Some new classes of integral trees with diameters 4 and 6. Australas. J. Comb. 21, 237-243 (2000)

11. Wang, L.G., Li, X.L.: Integral trees with diameters 4, 6 and 8. Australas. J. Comb. 25, 29-44 (2002)

12. Yuan, P.Z.: Integral trees of diameter 4. J. Syst. Sci. Math. Sci. 18(2), 177-181 (1998) 